\title{
Personalized medicine: biomarkers and companion diagnostics
}

Renato Bernardini ${ }^{1}$, Giovanni Gancitano ${ }^{2}$, Angela Balice ${ }^{1}$, Rosaria Di Mauro 1 , Giuseppina Cantarella ${ }^{1}$, Matteo Dionisi ${ }^{2}$

Department of Biomedical and Biotechnological Sciences, University of Catania, Italy

2 Medical \& Market Access Department, Roche Diagnostics SpA, Monza (MB), Italy

\begin{abstract}
Great expectations are bound to the current evolution of medicine to personalized medicine. Thanks to rapid advances in genomics and molecular biology, new markers can be revealed for the presence of or susceptibility to a disease, or response to treatment. On such markers, diagnostic tests can be based; companion diagnostics (CDx, often called In Vitro devices) are diagnostic tests "coupled" with a therapeutic drug, aimed at assessing its applicability to a specific class of patients. As well as exemplifying some already implemented CDx applications, the purpose of this article is to highlight potentials and problems of personalized medicine today. In particular, the opportunity is analyzed for the co-development of a new drug and its CDx, through a parallel base research. This approach is promoted by the regulatory agencies but, due to scientific and economic factors implicit in the process, it is taking-off slowly. Personalized medicine deserves to grow and to expand, first of all because it simultaneously promises to substantially improve patient care and to make big costs savings for healthcare systems. From this point of view, all stakeholders (diagnostics manufacturers, clinical testing laboratories, pharmaceutical firms, the Department of health, and other bodies) should talk to each other in order to support the advancement of personalized medicine.
\end{abstract}

\section{Keywords}

Personalized medicine, Companion diagnostics, Biomarkers

\section{INTRODUCTION}

Medical advances in diagnostics and therapeutics allow today to highlight new aspects of clinical practice, laying the foundations for the so-called "personalized medicine". Among the many definitions aimed at outlining the profile of "personalized health care", we would like to mention the one proposed by the European Commission in 2013: "a model of medicine that uses the molecular profile to individualize the correct therapeutic strategy for the right person at the right time, and/or to identify the predisposition to a certain disease and/or to allow for a timely and targeted prevention» [1].

The increasing use of target therapies supports the concept of "translational research", adapting to the therapeutic and clinical needs of each individual, especially within a setting of neoplastic and - more generally - chronic degenerative diseases [2].

In the future of personalized medicine, the role of Companion Diagnostics (CDx) seems indispensable. CDx are In Vitro Diagnostics (IVDs) which can select, within a population, those individuals who can benefit more from a certain treatment, and with a lower risk.

As such, CDx are a valuable guidance in clinical practice, and their accuracy is crucial for the success of the therapeutic choice.

The molecular characterization of the host microenvironment is therefore assuming an increasingly important role, next to histopathological typing, thus reinforcing the clinical value of the latter.

\section{ROLE IN CLINICAL PRACTICE}

The development of a CDx is based on the previous identification of specific biomarkers.

The clinical role of a CDx can be:

Corresponding author Giovanni Gancitano giovanni.gancitano@roche.com 
- diagnostic: aimed at an early identification of the disease;

- susceptibility/risk: aimed at identifying the risk of developing the disease;

- prognostic: aimed at identifying the evolution of the disease;

- predictive: aimed at identifying the response to a drug and/or its toxicity;

- surrogate: substitute for a clinical endpoint.

The introduction of biomarkers is profoundly changing the clinical testing of drugs, introducing new trial designs, such as the umbrella trial and the basket trial: in the first case, patients are enrolled on the basis of the tumor site and receive appropriate treatments for each of the biomarkers identified; in the second, subjects are enrolled on the basis of the molecular characteristics of the tumor, and therefore patients with tumors in different organs may be enrolled in the same treatment arm.

The use of IVD tests can identify the mutations of some biomarkers, and is therefore becoming a real opportunity for the implementation of therapeutic strategies for cancer patients and other patients.

The use of biomarkers in oncology considers both the characteristics of the tumor (cell changes, mutations) - in the perspective of the effectiveness of the drug - and the patient's characteristics (genetic makeup), against the risk of potential toxicity [3].

Below are some examples, used to emphasize how the use of an IVD test can conclusively affect the therapeutic, organizational, and economic choices:

1. EGFR (Epidermal Growth Factor Receptor) mutations are already predictive biomarkers for the targeted treatment of advanced NSCLC (Non-Small Cell Lung Cancer). Currently, the prescription of EGFR tyrosine kinase inhibitors (EGFR-TKIs) is related to the identification of a clinically relevant EGFR mutation in the DNA derived from the tumor tissue. In addition, it has been noticed that, in such tests, in $12.2 \%$ of cases the mutational status still could not be assessed [4]. The analysis of the DNA derived from plasma can then be an important alternative even when, under certain conditions, the use of tissue is unfeasible, or too risky. In addition, liquid biopsy - already validated in many clinical trials - is a non-invasive, reproducible method, also obtainable from patients with severe morbidity. In this regard, the results of a recent study with osimertinib - an inhibitor of EGFR T790M mutation-related tyrosine kinases - support the use of the COBAS ${ }^{\circledR}$ test to detect EGFR mutations through plasma samples [4]. One of the many advantages of this test (as however is also the case of other CDx) is the swiftness of its execution. In June 2016, the FDA (Food and Drug Administration) therefore approved the COBAS ${ }^{\circledR}$ test as CDx (EGFR mutation test v2, Roche Molecular Systems, Inc.), to be used on liquid biopsy samples. EGFR mutation testing for the selection of treatments in NSCLC patients were developed following the introduction of first- and second-generation EGFR TKIs. Despite the fact that the analysis of the tissue biomarkers still represents the gold standard, it should be noted, however, that up to $30 \%$ of the patients with advanced NSCLC cannot benefit from it, due to a lack of suitable tissue [4]. In particular, with third-generation EGFR TKIs (osimertinib), it's not always possible to execute the Exon 20 T790M mutation test using tissue samples; in this case, liquid biopsies are a great opportunity.

2. Another recent study presented a CDx able to accurately and quickly identify NSCLC $\mathrm{ALK}+$ (Anaplastic Lymphoma Kinase) patients, who may benefit from the treatment with crizotinib [5].

3. The success of Herceptin in treating breast cancer still lies in the CDx ability to identify the subpopulation responsive to the MAb, namely the HER2+. The above example is a demonstration of how a careful stratification of the population can be decisive for the clinical efficacy of a drug, limiting its toxicity (cardiotoxicity - in the case of Herceptin) and containing the healthcare spending. The CDx for Herceptin (Herceptest) was the first companion diagnostic to enter the market, in 1998; then, an increase in the use of CDx led to an improvement in the therapeutic performance also in the treatment of other cancers: colorectal, lung, chronic myeloid leukemia, B-Cell non-Hodgkin's lymphoma.

\section{DEVELOPMENT PROCESS}

Companion diagnostics were officially defined in 2014 by the FDA as a "medical device that provides functional information for a safe and effective use of a drug or a biotechnological product" $[6,7]$.

Recently, also EMA (European Medicines Agency) decided to investigate the issue of $\mathrm{CDx}$, realizing the need for a co-development of the $\mathrm{CDx}$ and the related drug, in order to avoid the documentation gaps that may be incurred during a separate development [8]. 
Given the multiplicity of functions which characterize the biomarker and its related CDx, the technical requirements during the setting of the tests will vary, depending on the stage of development and use of the biomarker, resulting in timing issues related to the development of the CDx and the relative drug.

Despite everything, the co-development process proved to be less costly than the "postapproval" method, and therefore it is desirable to proceed with a parallel basic research that takes concurrently into account the development of a drug and its CDx [8,9]. Simultaneously with the preclinical development of the drug, feasibility studies are conducted on the selected biomarkers [10]. In the clinical stage, during Phase I, candidate markers in humans are assayed [11], while during Phase II - which is parallel to the analytical validation of the biomarkers - the clinical criteria for stratification are defined. During Phase III, such stratification is further validated and, on the basis of the outcomes of the trial, new considerations are made on the label.

This leads to the validation of CDx - and the simultaneous approval of the drug and the relevant IVD - by the Regulatory Authorities.

\section{REGULATORY ASPECTS}

From a regulatory point of view, the current situation is very delicate, because the Regulatory Authorities, which in fact continue to allow the use of low performance tests, have to deal with the need to introduce validated tests that meet the therapeutic expectations of increasingly more numerous and expensive drugs.

Such evidence cannot be ignored for a long time, in view of the increasing complexity of the therapeutic scenario, driven by the continuous discovery of new molecular targets for an optimal therapy, aimed as much as possible at meeting the needs of selected subpopulations within the same nosological framework.

Therefore, if on the one hand the introduction of CDx developed concurrently with a specifically targeted drug raises the hopes of obtaining a personalized therapy, on the other the regulatory situation both in the US and in Europe needs to be improved. In fact, the global regulatory framework concerning the molecular diagnostic tests - including CDx - is currently rather fragmented and inconsistent and there are still issues regarding the quality, safety and effectiveness of such tests [12], with few regulatory initiatives undertaken to date in an attempt to accelerate the development of biomarkers.

Despite a number of initiatives undertaken by Regulatory Authorities, there is still a clear need for greater efforts, possibly supported by the creation of technical committees tasked with formulating hypotheses and propositions aimed at making the simultaneous marketing of high performance diagnostic tests compatible with that of the drugs whose need and market presence are more obvious every day.

\section{CRITICAL ISSUES}

Despite the results achieved so far in the field of personalized therapies, a significant increase in the development of CDx has not occurred, yet; in fact, over the last 17 years, only 23 CDx were approved in the US, for 11 personalized therapies and 7 first-line indications [13]. Several are the causes of the slow growth of CDx, and they are all due to the difficulty of a CDx-drug co-development. In this respect, in light of the personalized medicine implications, also the pharmacoeconomic model must be radically reconsidered [9].

It is likely that the sheer amount of obstacles identified so far in the development process of CDx has limited private and public investment in this field. In 2014 in fact, the FDA issued guidelines that promoted CDx-drug co-development up to the marketing step. This regulatory constraint suggested by the FDA affects the economic factor related to the final product, and therefore influences the amount of products marketed and prescribed. It is indeed difficult to resolve the alignment between manufacturers of diagnostic and pharmaceutical companies, because of the complexity of the co-development process, and also due to the fact that, to date, there is an objective lack of systematic analytical studies aimed at understanding the changes that would be necessary to implement within the current system, and that the market orientation now requires [9].

Considering the clinical relevance of CDx, but also the aforementioned limitations, one can clearly outline the opportunity to promote a systematic analysis of the already existing data, in order to identify the drivers and any limitations, and to characterize the areas in which to intensify research. 
The challenge in the research field will therefore being able to identify the most important molecular, diagnostic, prognostic and predictive targets, capable to predict therapeutic responsiveness, in order to select eligible patients [14,15].

Also in light of the recent regulatory focus on the CDx, it is evident that this technology - through a correct identification of specific biomarkers and specific patient populations - can also be considered a major tool for the control of the market of high-effectiveness and high-cost drugs. The development of CDx appears therefore based on the identification of biomarkers, which thereby make it possible to select the best treatment for each single patient. From a pharmaceutical development perspective, the CDx can contribute to the success of the process of approval and marketing of innovative drugs, which however - in addition to a greater therapeutic benefit - may also result in a high risk, in case of misuse.

The accuracy of the CDx is critical in order to achieve good clinical results and avoid any waste of resource: there is evidence in the literature of how an inaccurate use of CDx leads to therapeutic failures and substantial expenditure; this is the case, for example, of the tests for the detection of EFGR mutations in patients with metastatic NSCLC [12]. Here, the clinical trial design ought to be thoroughly reconsidered: biology is breaking down diseases into increasingly smaller subsets, for each of which viable therapies will have to be identified [16].

The use of CDx presents considerable limitations, due to the many obstacles that still must be overcome before this method can be established as an indispensable support to clinical practice.

One of the main problems is the objective finalistic limits that characterize the evaluation of the biomarkers, which are due to the likelihood of bias (false positives, false negatives) in the characterization of the same through currently available methods. It should also be considered that the possibility to evaluate more than one biomarker in a tissue entails the need to have substantial quantities of the sample, which is often difficult in clinical practice; this is therefore a further limit to the increase in the use of CDx [3]. All this significantly affects the therapeutic appropriateness - and thus the clinical outcome - canceling part of the progress achieved through genomics and proteomics, aimed at the full realization of a "Precision Medicine": having adequate amounts of a sample is, in fact, essential in order to make a genomic/ proteomic characterization, which in turn is essential for a targeted selection of the drug.

The literature, in fact, shows how the identification of a single therapeutic target is not sufficient to predict with accuracy the effectiveness of a drug. By way of example, we now know that patients eligible to anti-EGFR therapy may not benefit from it, if the tumor also presents RAS mutations: this is the case of certain colon-rectum and lung tumors (KRAS, NRAS); another example is represented by patients with $\mathrm{BCR} / \mathrm{ABL}+$ leukemia who, in the presence of ABL-kinase mutations, may not respond to imatinib, therefore necessitating a second- or third-line therapy with TKIs.

There are also important economic aspects to consider in the management of CDx and, more generally, in the high-performance diagnosis of chronic degenerative diseases. The ratio between the average cost of an anticancer therapy (e.g. ALK inhibitors for NSCLC) and the cost of an assay with the relevant diagnostic can be 1000:1. It would therefore be possible to achieve large savings in treatment costs by excluding those tumor variants which are potentially refractory to treatment, identified with an optimized diagnostic algorithm [17]. In fact, the trend of the world Health Services to reimburse high-complexity laboratory tests is gradually declining, also as a result of the remodeling of the reimbursement methodology implemented by the National and Regional Authorities which, in an attempt to maintain the sustainability of the system, consider more favorably low-price diagnostic tests which involve, among other things, the use of a smaller amount of manpower. On the issue of reimbursement, payers, pharmaceutical companies and manufacturers of CDx will have to explore new risk-sharing patterns [18].

\section{FUTURE CHALLENGES}

In the face of financial straits, the growing clinical need to replace traditional diagnostic tests with new generation ones - for a better and more efficient definition of the biological profile of the disease, and thus of the possibility of creating personalized therapies - is becoming preponderant. The adoption of a harmonized platform of CDx validated by the Regulatory Agencies is therefore desirable.

Despite the strong will on the part of the Regulatory Authorities to harmonize the CDx co-development procedure, no economic strategy has yet been implemented to achieve this objective [13]. 
Ideally, the use of new generation CDx should be extended to all patients with a diagnosis of cancer and/or chronic degenerative disease, in order to select them and include them in the appropriate subpopulation for treatment; however, in the perspective of a healthcare savings, it would be appropriate - at least initially - to restrict this type of diagnostic approach to the patients who are refractory to other treatments. This procedure would have the dual advantage of providing a benefit to patients with little or no therapeutic alternatives, and to expand the number of evidence in the real life setting. All this would facilitate the progressive validation of methodologies based on clinical data, and their gradual application to increasingly large populations, after the due regulatory considerations on an informed cost management, thereby making the healthcare systems availability compatible with the need to cover as broadly as possible - in order to improve it - the need for a more accurate "centering" of a particular pathological condition.

In order for the CDx resource to be exploited to the fullest, it is necessary that all the actors of the system (manufacturers of diagnostics, laboratories performing the tests, pharmaceutical companies, scientific societies, regulatory agencies, ministries and others) talk to each other, to develop guidelines specifically aimed at an appropriate use of the CDx.

Naturally, this balance is very delicate, and its realization seems neither easy nor obvious, both in consideration of the time required for the Regulatory Authorities to carefully assess the quality of the test development process and its actual value in the context of clinical trials, and in view of the rapid evolution of technology. The path to a synchronized and shared agreement is therefore not yet clear, and goes through the evaluation of the test and the tools to implement it, the assessment of the quality control criteria, the standardization of procedures and the evaluation of the actual clinical relapses. Although at the moment we are in a phase where the complex regulatory system rightly requires a balanced and proportionate evaluation of the innovation, also with regard to $\mathrm{CDx}$, the possibility to associate the appropriate therapy, individualized for a specific disease, does not appear, from a technical point of view, too far away.

In this perspective, particularly in oncology, it is now essential that future clinical studies are designed so as to match the therapy to a specific molecular makeup $[18,19]$.

As mentioned above, while current CDx have often been developed only after the introduction of the drug on the market, Regulatory Agencies are now showing a preference for the co-development of drug and CDx. The main challenge of the future may indeed be the creation of a single regulatory system for all medicinal products, which addresses both aspects, the drug and the $\mathrm{CDx}$, so as to submit them to the authorization procedure combined as a single product.

\section{CONCLUSIONS}

The progressive implementation of personalized medicine in the healthcare systems will be of great interest, from different perspectives: for patients, who will benefit from better results in response to targeted therapies (i.e. personalized, or stratified); for health professionals, who will face a lower incidence of adverse reactions; for payers, who will be able to improve the allocation of economic resources, by investing in treatments with a high response rate and a better cost-effectiveness. All this will take place in parallel to a growing sustainability of the health system.

Whether the answer to chronic-degenerative - and in particular neoplastic - diseases will be significantly more effective than it is at present, with a primary benefit to the patient, the healthcare system and, therefore, the entire society, will depend on the effectiveness of the dialogue between the actors of the system and the reasonable progressive application of diagnostic methods that, hopefully, sooner or later will become an indispensable support to quality care.

\section{Funding}

This research was made possible by an educational grant from Roche Diagnostics S.p.A.

\section{Conflicts of interest}

$\mathrm{RB}, \mathrm{AB}, \mathrm{RDM}$ and $\mathrm{GC}$ declare that they have no conflicts of interest in this research. GG and MD are full-time employees of Roche Diagnostics S.p.A. 


\section{REFERENCES}

1. European Commission 25.10.2013 SWD(2013) 436 final: Commission staff working document: Use of '-omics' technologies in the development of personalised medicine. Available at https://ec.europa.eu/research/health/pdf/2013-10_personalised_medicine_en.pdf (last accessed December 2017)

2. President's Council of Advisors on Science and Technology. Priorities for Personalized Medicine. September 2008. Available at https://www.hsdl.org/?view\&did=234678 (last accessed December 2017)

3. Rodriguez-Antona C, Taron M. Pharmacogenomic biomarkers for personalized cancer Treatment. J Intern Med 2015; 277: 201-17; https://doi.org/10.1111/joim.12321

4. Malapelle U, Sirera R, Jantus-Lewintre E, et al. Profile of the Roche COBAS ${ }^{\circledR}$ EGFR mutation test v2 for non-small cell lung cancer. Expert Review of Molecular Diagnostics 2017; 17: 209-15; https://doi.org/10.1080/14737159.2017.1288568

5. Thorne-Nuzzo T, Williams C, Catallini A, et al. A Sensitive ALK Immunohistochemistry Companion Diagnostic Test Identifies Patients Eligible for Treatment with Crizotinib. $J$ Thorac Onco 2016; 12: 804-13; https://doi.org/10.1016/j.jtho.2017.01.020

6. U.S. Food \& Drug Administration. Companion Diagnostics. Available at https://www.fda. gov/medicaldevices/productsandmedicalprocedures/invitrodiagnostics/ucm $407297 . \mathrm{htm}$ (last accessed December 2017)

7. Donovan MJ, Cordon-Cardo C. Implementation of a Precision Pathology Program Focused on Oncology-Based Prognostic and Predictive Outcomes. Mol Diagn Ther 2017; 21: 11523; https://doi.org/10.1007/s40291-016-0249-5

8. European Medicines Agency. Concept paper on predictive biomarker-based assay development in the context of drug development and lifecycle. 2017. EMA/CHNP/800914/2016. Available at http://www.ema.europa.eu/docs/en_GB/document_library/Scientific_guideline/2017/07/WC500232420.pdf (last accessed December 2017)

9. Degeling K, Koffijberg H, IJzerman MJ. A systematic review and checklist presenting the main challenges for health economic modeling in personalized medicine: towards implementing patient-level models. Expert Rev Pharmacoecon Outcomes Res 2017; 17: 17-25; https://doi.org/10.1080/14737167.2017.1273110

10. Wulfkuhle JD, Spira A, Edmiston KH, et al. Innovations in Clinical Trial Design in the Era of Molecular Profiling.- Molecular Profiling: Methods and Protocols, Methods in Molecular Biology. Vol 1606. New York: Humana Press, 2017

11. Dearden S, Brown H, Jenkins S, et al. EGFR T790M mutation testing within the osimertinib AURA Phase I study. Lung Cancer 2017; 109; 9-13; https://doi.org/10.1016/j. lungcan.2017.04.011

12. Cheng MM, Palma JF, Scudder S, et al. The Clinical and Economic Impact of Inaccurate EGFR Mutation Tests in the Treatment of Metastatic Non-Small Cell Lung Cancer. J Pers Med 2017; 7; https://doi.org/10.3390/jpm7030005

13. Luo D, Smith JA, Meadows NA. A Quantitative Assessment of Factors Affecting the Technological Development and Adoption of Companion Diagnostics. Front Genet 2016; 6: 357; https://doi.org/10.3389/fgene.2015.00357

14. Tímár J, Ladányi A. Predictive markers of immunotherapy of cancer, practical issues of PD-L1 testing. Magy Onkol 2017; 61: 158-66

15. Nagy Z. Biomarkers in solid tumors. Magy Onkol 2013; 57: 56-62

16. Berry DA. The Brave New World of clinical cancer research: Adaptive biomarker-driven trials integrating clinical practice with clinical research. Mol Oncol 2015; 9: 951-9; https:// doi.org/10.1016/j.molonc.2015.02.011

17. Nenadić I, Staber J, Dreier S, et al. Cost Saving Opportunities in NSCLC Therapy by Optimized Diagnostics. Cancers 2017; 9: 88; https://doi.org/10.3390/cancers9070088

18. Brüggenjürgen B, Kornbluth L, Ferrara JV, et al. Clinical and health economic challenges of personalized medicine. Bundesgesundheitsblatt Gesundheitsforschung Gesundheitsschutz 2012; 55: 710-4; https://doi.org/10.1007/s00103-012-1479-2

19. Renfro LA, An M-W, Mandrekar SJ. Precision oncology: A new era of cancer clinical trials. Cancer Lett 2017; 387: 121-6; https://doi.org/10.1016/j.canlet.2016.03.015 